\title{
Expression of decorin in intestinal tissues of mice with inflammatory bowel disease and its correlation with autophagy
}

\author{
HUAZHOU ZHAO ${ }^{1,2}$, HONGQING XI $^{1}$, BO WEI ${ }^{1}$, AIZHEN CAI $^{1}$, TING WANG $^{3}$, \\ YI WANG ${ }^{1,2}$, XUDONG ZHAO ${ }^{1}$, YANJING SONG ${ }^{1}$ and LIN CHEN $^{1}$ \\ ${ }^{1}$ Department of General Surgery, General Hospital of Chinese People's Liberation Army, Beijing 100853; \\ ${ }^{2}$ Department of General Surgery, 309 Hospital of Chinese People's Liberation Army, Beijing 100091; \\ ${ }^{3}$ Medical Administration Division of Beijing Chaoyang Hospital, Beijing 100020, P.R. China
}

Received August 6, 2015; Accepted September 15, 2016

DOI: $10.3892 / \mathrm{etm} .2016 .3908$

\begin{abstract}
The aim of this study was to investigate the expression of decorin $(\mathrm{DCN})$ in the intestinal tissues of mice with inflammatory bowel disease (IBD) and its correlation with autophagy. The IBD mouse model was created by intrarectal injection of trinitrobenzene sulfonic acid. The pathology of colon tissues of the mice was examined using hematoxylin and eosin staining. Expression of DCN and the proteins associated with autophagy was detected using immunohistochemistry. Normal human colon mucosal epithelial cells (NCM460 cells) were transfected with DCN expression plasmid and the expression of DCN and autophagy-associated proteins was detected by western blot analysis. Cell apoptosis was studied using an Annexin $\mathrm{V}$ apoptosis detection assay and intracellular autophagosomes were observed using electron microscopy. The IBD mouse model was successfully established. Thickening, edema and inflammatory cell infiltration of the intestinal wall were observed in the IBD mice. The expression of DCN as well as the autophagy-associated proteins beclin 1 and LC3B, was increased in the intestinal tissues of the IBD mice. Furthermore, in the NCM460 cells transfected with DCN, the expression of beclin 1 and LC3B was upregulated, while p62 expression was downregulated. Intracellular autophagosomes were increased and apoptosis was decreased in the cells with DCN overexpression. Inhibition of autophagy reversed the effects of DCN on apoptosis. Therefore, DCN is able to induce autophagy and protect intestinal cells during the occurrence and development of IBD.
\end{abstract}

Correspondence to: Dr Bo Wei or Dr Lin Chen, Department of General Surgery, General Hospital of Chinese People's Liberation Army, 28 Fuxing Road, Beijing 100853, P.R. China

E-mail: weibobj301@sina.com

E-mail: sdc1678@126.com

Key words: decorin, inflammatory bowel disease, autophagy

\section{Introduction}

Inflammatory bowel disease (IBD) is a group of common chronic inflammatory gastrointestinal disorders, including Crohn's disease and ulcerative colitis $(1,2)$. IBD usually occurs in young adults and is closely associated with inheritance, infection and immune function (3). IBD is harmful to health with characteristics including a high recurrence rate, high canceration rate and poor prognosis $(4,5)$. Further investigation of the molecular mechanisms of IBD may provide valuable information for the treatment of IBD.

The pathogenesis of IBD is complex. Intestinal mucosal inflammation can lead to sustained and irreversible damage to the digestive tract and produce serious clinical consequences (6). A variety of inflammatory mediators such as interleukin (IL)-4, IL-1, tumor necrosis factor and transforming growth factor (TGF)- $\beta$, as well as several signal pathways such as the nuclear factor- $\kappa \mathrm{B}$ signaling pathway, participate in the damage of the digestive tract (7-9). Immunosuppressant therapy is an important method for the treatment of IBD, as it is able to relieve intestinal inflammation and results in significant treatment efficiency (10). Autophagy is an important cellular pathway for the maintenance of homeostasis. It degrades damaged organelles, misfolded proteins and damaged DNA to provide energy that allows the cells to respond to adverse environments $(11,12)$. Autophagy is closely associated with inflammation (13) and is induced by a variety of inflammatory factors. Conversely it is also able to downregulate inflammation (14). However, the exact molecular mechanisms require further investigation.

Decorin $(\mathrm{DCN})$ is a small leucine-rich proteoglycan with glycosaminoglycan chains attached to a core protein and is one of the important components of the extracellular matrix (15). Abnormal expression of DCN or alteration of the structure of DCN correlates closely with a variety of pathological processes including inflammation and cancer $(16,17)$. DCN has a number of biological functions, including inhibition of the proliferation, invasion and metastasis of a variety of tumors and is a potential target protein for cancer therapy $(18,19)$. In inflammation, DCN expression negatively correlates with TGF-1 and IL-1, and positively correlates with IL-4, indicating that DCN is involved in the occurrence and development of 
inflammation (20). Currently, the expression and function of DCN in IBD remains unclear. In the present study, IBD mouse models were produced and the expression of DCN, as well as autophagy-associated proteins in the intestinal tissues of the IBD mice was examined. To further investigate the biological functions of DCN in IBD, the effects of DCN on autophagy regulation in normal human colon mucosal epithelial cells (NCM460 cells) were studied.

\section{Materials and methods}

Reagents. M3 medium was purchased from Gibco (Thermo Fisher Scientific, Inc., Waltham, MA, USA). 3-Methyladenine (3-MA) was purchased from Sigma-Aldrich (Merck Millipore, Darmstadt, Germany). Fluorescein isothiocyanate (FITC) Annexin V Apoptosis Detection kit I was purchased from BD Biosciences (Franklin Lakes, NJ, USA). Rabbit anti-human/mouse DCN (\#P07585) and beclin 1 (\#Q14457) antibodies, and mouse anti-human/mouse GAPDH (\#O14556) antibody were purchased from Bioworld Technology, Inc. (St. Louis Park, MN, USA). Rabbit anti-human/mouse p62 antibody (\#8025) was purchased from Cell Signaling Technology, Inc. (Boston, MA, USA). Rabbit anti-human/mouse LC3B antibody (\#AL221) was purchased from Beyotime Institute of Biotechnology (Beijing, China). DCN expression plasmid (pEGFP-N1-DCN) was purchased from Ruino (Guangzhou, China). Lipofectamine 2000 transfection reagent was purchased from Invitrogen (Thermo Fisher Scientific, Inc.).

IBD mouse model. The study was approved by the Ethics Committee of the General Hospital of Chinese People's Liberation Army (Beijing, China). A total of 60 healthy adult male BALB/c mice weighing $21 \pm 1.34 \mathrm{~g}$ were purchased from Chengdu DOSSY Experimental Animal Co., Ltd. (Chengdu, China) and kept under specific-pathogen-free conditions. The animals were housed under a 12-h light/dark cycle and allowed ad libitum access to rodent chow and water. The room temperature was maintained at $22^{\circ} \mathrm{C}$ and $50 \%$ relative humidity. The mice were randomly divided into three groups ( $n=20$ per group), namely the normal group, the control group and the IBD group. In the IBD group, the mice were anesthetized with pentobarbital $(50 \mathrm{mg} / \mathrm{kg}$; Shanghai Chemical Reagent Company, Shanghai, China) after defecation and kept with the head down in a vertical position. A polyethylene pipe with a diameter of $0.2 \mu \mathrm{m}$ was inserted into the colon $(4 \mathrm{~cm}$ proximal to the anus) of each mouse and a dose of $200 \mathrm{mg} / \mathrm{kg}$ trinitrobenzene sulfonic acid (TNBS; Sigma-Aldrich) solution in $50 \%$ ethanol was injected intrarectally. After that, the mice were fed normally. TNBS administration was performed once a day for 7 days and $24 \mathrm{~h}$ after the last administration, the mice were sacrificed after anesthetization with $50 \mathrm{mg}$ / $\mathrm{kg}$ pentobarbital. In the control group, the mice received only $50 \%$ ethanol. In the normal group, the mice were kept under normal conditions without treatment. The colon tissues of the mice were removed and fixed with formaldehyde or stored in liquid nitrogen.

Cell culture. NCM460 cells were purchased from American Type Culture Collection (Manassas, VA, USA). The cells were cultured in M3 medium supplemented with $10 \%$ fetal bovine serum (FBS; Gibco). The media was changed every other day and the cells were passaged using trypsin digestion on reaching $80-90 \%$ confluence.

Hematoxylin and eosin $(H \& E)$ staining. H\&E staining was performed on the colon tissues of the mice. Briefly, the colon tissues were fixed with $10 \%$ formaldehyde for $24 \mathrm{~h}$, rehydrated in graded alcohols, hyalinized with xylene, embedded in paraffin and cut into $2-\mu \mathrm{m}$ tissue sections. The tissue sections were dewaxed in xylene, stained with hematoxylin, stained with eosin, rehydrated in graded alcohols and hyalinized with xylene. After that, the sections were mounted with neutral gum and observed using an optical miscroscope (BX50; Olympus Corporation, Tokyo, Japan.

Immunohistochemistry. The expression of DCN, beclin 1 and $\mathrm{LC} 3 \mathrm{~B}$ in the colon tissues of the mice was detected using immunohistochemistry. The colon tissues were fixed with $10 \%$ formaldehyde, embedded in paraffin and cut into $4-\mu \mathrm{m}$ sections. The sections were dewaxed and rehydrated in graded xylene and alcohols. The sections were then incubated with $3 \% \mathrm{H}_{2} \mathrm{O}_{2}$ at room temperature for $10 \mathrm{~min}$ to inactivate endogenous peroxidase and processed for antigen retrieval using microwave heating at $400 \mathrm{~W}$ for $15 \mathrm{~min}$. Nonspecific binding was blocked with $10 \%$ goat serum (diluted with PBS) at room temperature for $10 \mathrm{~min}$. The sections were incubated with anti-DCN antibody (1:200), anti-beclin 1 antibody (1:200) and anti-LC3B antibody, respectively, at room temperature for $1 \mathrm{~h}$, then with goat anti-rabbit $\operatorname{IgG~H\& L}$ biotinylated secondary antibody(1:200; \#ab97049; Abcam, Cambridge, MA, USA) at $37^{\circ} \mathrm{C}$ for $30 \mathrm{~min}$. The sections were then developed with 3,3'-diaminobenzidine chromogenic reagent. Finally, the sections were counterstained with hematoxylin for $\sim 30 \mathrm{sec}$. Following hydrochloric acid differentiation and hyalinization with xylene, the sections were mounted with neutral gum and observed under an optical microscope.

Positive cells were defined as cells with brown staining in the cytoplasm or on the cell membrane. For each section, five fields were randomly taken under a high power field (HPF). The positive cells and negative cells were counted in each field. The positive rate of each field was the percentage of the ratio of the number of positive cells to the number of total cells (the sum of positive and negative cells). The positive rate of each tissue section was expressed as the mean of the positive rate of the five fields. The expression of the proteins was scored according to both the positive rate and the degree of staining. The positive rate was scored as following: $0 \%$, score $0 ; 1-25 \%$, score $1 ; 26-50 \%$, score 2 and $51-100 \%$, score 3 . The degree of staining was scored as following: Without staining, score 0 ; with light yellow staining, score 1; with light brown staining, score 2 and with brown staining, score 3. The final score of the expression of the proteins was obtained by multiplying the score of the positive rate with the score of the degree of staining and was graded as following: Score 0 or 1 , negative; score 2 or 3 , weakly positive; score 4-6, positive and score $>6$, strongly positive.

Transmission electron microscopy observation. NCM460 cells were seeded into $10-\mathrm{mm}$ culture plates. After culturing for $24 \mathrm{~h}$, the culture medium was discarded and cells were 
washed with PBS twice. Then, the cells were fixed in $2.5 \%$ glutaric dialdehyde for $30 \mathrm{~min}$ at $4^{\circ} \mathrm{C}$. Cells were collected into 1.5-ml EP tubes by scraping. Then, $2.5 \%$ glutaric dialdehyde was added and incubated at $4^{\circ} \mathrm{C}$ overnight. The subsequent procedures were performed by Shanghai Fucheng Biological Technology Co. Ltd. (Shanghai, China).

Western blot analysis. The colon tissues of the mice, ground into a powder in liquid nitrogen, were lysed with radioimmunoprecipitation assay buffer containing phenylmethanesulfonyl fluoride. The lysates were subjected to electrophoresis on $11 \%$ SDS-PAGE gels. The proteins were then transferred to poly(vinylidene fluoride) membranes for western blot analysis. After blocking with 5\% non-fat milk for $1 \mathrm{~h}$ at room temperature, the membranes were incubated overnight with anti-DCN, anti-beclin 1, anti-LC3B antibody, anti-p62 (all 1:1,000) or anti-GAPDH $(1: 10,000)$ antibodies at $4^{\circ} \mathrm{C}$ overnight. After washing, the membrane was incubated with goat anti-mouse (1:3,000; \#ab6789) and anti-rabbit (1:4,000; \#ab6721) horseradish peroxidase-conjugated secondary antibody (Abcam) at room temperature for $1 \mathrm{~h}$. Bound antibodies were detected using an enhanced chemiluminescence kit (Amersham; GE Healthcare, Little Chalfont, UK). The mean normalized optical density (OD) of the DCN, beclin 1, LC3B or p62 protein band relative to the OD of GAPDH band from the same sample was calculated using Quantity One software, version 4.62 (Bio-Rad Laboratories, Inc., Hercules, CA, USA).

Cell transfection. NCM460 cells in the logarithmic growth phase were seeded at $2 \times 10^{5}$ cells/well in 24 -well plates. On the next day, NCM460 cells were transfected with $1 \mu \mathrm{g}$ DCN expression plasmid (pEGFP-N1-DCN) using Lipofectamine 2000 according to the manufacturer's instructions. At $48 \mathrm{~h}$ after transfection, the cells were harvested and lysed for western blot analysis as described above. Cells without transfection or transfected with empty plasmid were used as controls.

Apoptosis assays. NCM460 cells were transfected with DCN expression plasmid as above described. The autophagy inhibitor 3-MA (5 $\mu \mathrm{M})$ was added and incubated for $24 \mathrm{~h}$. Then, cells were cultured with fresh glucose-free medium in a three-gas incubator $\left(5 \% \mathrm{CO}_{2}, 1 \% \mathrm{O}_{2}\right.$ and $\left.94 \% \mathrm{~N}_{2}\right)$ with saturated humidity. After $24 \mathrm{~h}$, cells were collected for apoptosis analysis. Apoptosis assays were performed using the FITC Annexin V Apoptosis Detection kit I according to the manufacturer's protocol. Cells that are in early apoptosis are Annexin $\mathrm{V}$ positive and propidium iodide (PI) negative, cells that are in necrosis are PI positive and Annexin V negative, and cells that are in late apoptosis are Annexin V and PI positive.

Statistical analysis. All data were processed using the SPSS version 17.0 statistical package (SPSS, Inc., Chicago, IL, USA). Data are presented as means \pm standard deviation. Statistical significance was determined using the Student's t-test. $\mathrm{P}<0.05$ was considered to indicate a statistically significant result.

\section{Results}

Successful modeling of IBD in mice. The IBD mouse model was generated by intrarectal injection of TNBS. The pathology of colon tissues in the mice was examined using $H \& E$ staining. In the normal group and the control group, the mice were in good spirits with normal weight and stools. The hair of the mice was smooth and flat. In the IBD group, the mice remained alive throughout the whole experiment, until sacrifice. However, they were lethargic with reduced physical activity and yellow mushy stools. In some mice, the stools contained mucus, blood and pus and their hair stood on end. H\&E staining showed mucosal erosion, inflammatory cell infiltration, thickening of the intestinal wall and ulceration in the colon tissues of mice in the IBD group but not in the normal group (Fig. 1).

Altered expression of DCN and autophagy-associated proteins in the intestinal tissues of IBD mice. To investigate the expression of DCN, as well as autophagy-associated proteins in the intestinal tissues of IBD model mice, immunohistochemical assays and western blot analysis were performed. As shown in Fig. 2, in the IBD group, DCN expression could be observed in the cytoplasm and nucleus of all cells in the intestinal tissue. The strong positive rate was $48 \%$. In the normal group and the control group, DCN expression was negative or weakly positive. The difference was statistically significant $(\mathrm{P}<0.05)$. The positive rates of autophagy-associated proteins beclin 1 and LC3B in the IBD group were significantly higher than those in the control group $(\mathrm{P}<0.05)$.

The expression levels of DCN, beclin 1, LC3B and p62 in the intestinal tissues of the mice were also examined using western blot analysis. As shown in Fig. 3, there were no significant differences in the expression of $\mathrm{DCN}$, beclin $1, \mathrm{LC} 3 \mathrm{~B}$ and p62 between the normal group and the control group. The expression of DCN, beclin 1 and LC3B was significantly higher in the IBD group than in the normal group $(\mathrm{P}<0.05)$. The expression of p62 was significantly lower in the IBD group than in the normal group $(\mathrm{P}<0.05)$. These results suggest that autophagy is induced in the intestinal tissues of the mice with IBD.

Increased expression of autophagy-associated proteins in NCM460 cells transfected with DCN expression plasmid. To further investigate the correlation of the expression of DCN with autophagy-associated proteins, NCM460 cells were transfected with DCN expression plasmid, and the expression of DCN and autophagy-associated proteins was detected using western blot analysis. As shown in Fig. 4, compared with NCM460 cells without transfection and NCM460 cells transfected with empty plasmid, the expression of DCN was significantly increased in the cells transfected with DCN expression plasmid $(\mathrm{P}<0.05)$. The expression of beclin 1 and LC3B was also significantly increased in the cells transfected with DCN expression plasmid $(\mathrm{P}<0.05)$. There were no significant differences in the expression of p62 between the cells transfected with DCN expression plasmid and the cells without transfection or transfected with empty plasmid. These results indicate that DCN may promote autophagy.

Increased autophagy in NCM460 cells transfected with DCN expression plasmid. To investigate the effects of DCN on autophagy, NCM460 cells were transfected with DCN expression plasmid and intracellular autophagosomes were observed using transmission electron microscopy. As shown in Fig. 5, 
A

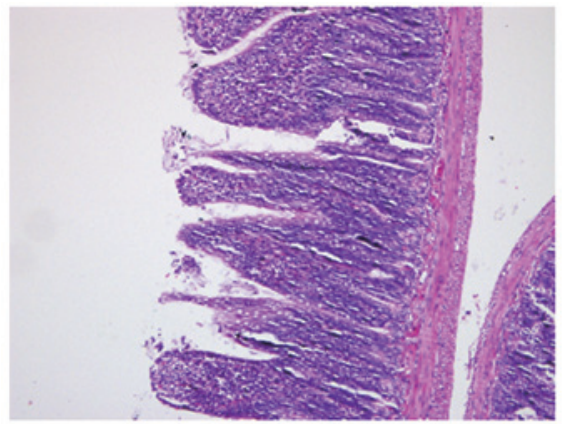

B

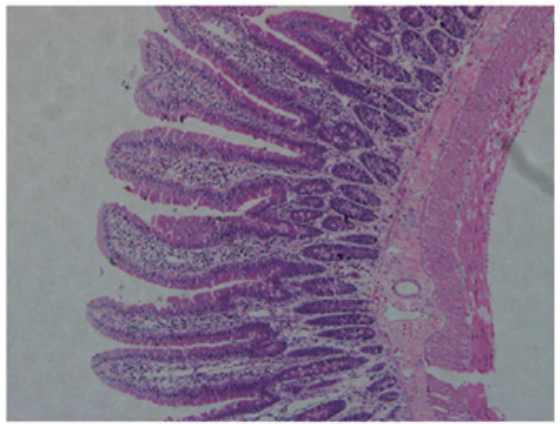

$\mathbf{C}$

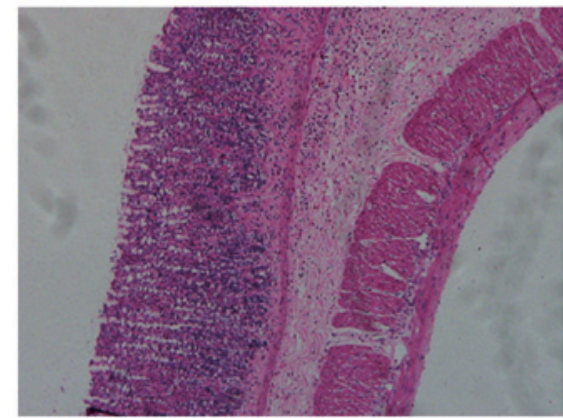

Figure 1. Histopathological observation of colon tissues (magnification, x200) using hematoxylin and eosin staining. Representative images of the colon tissues are shown. (A) In the normal group, there was no thickening of the intestinal wall, congestion or inflammatory cell infiltration. (B) In the control group, there was minor hyperplasia of the glands, slight thickening of the intestinal wall and a little inflammatory cell infiltration. (C) In the inflammatory bowel disease group, there was notable thickening of the intestinal wall, damaged glands, evident congestion and inflammatory cell infiltration throughout the intestinal wall.

A

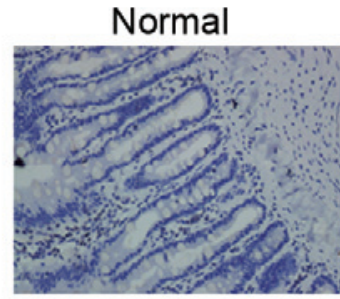

B

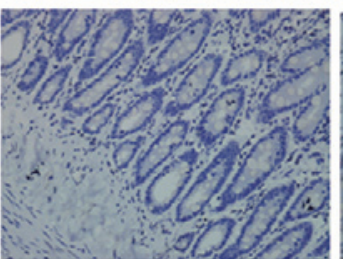

C

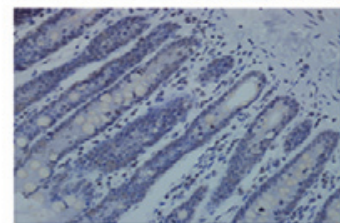

Control
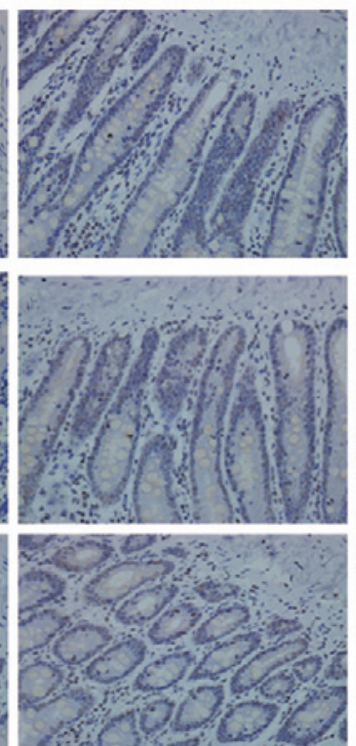

IBD
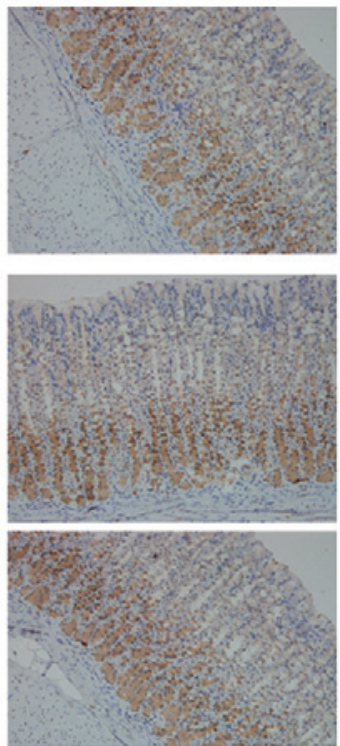

Figure 2. Expression of DCN and autophagy-associated proteins in the intestinal tissues detected using immunohistochemical assay (magnification, x200). Representative immunocytochemical staining results of (A) DCN, (B) beclin 1 and (C) LC3B are shown. Positive cells were stained brown. (A) DCN expression was negative in the normal group, weak in the control group and significantly increased in the IBD group. (B) In the normal and control groups, beclin 1 expression was negative or weakly positive. There was no significant difference in beclin 1 expression between these two groups. In the IBD group, beclin 1 expression significantly increased. (C) In the normal and control groups, LC3B expression was negative or weakly positive. There was no significant difference in LC3B expression between these two groups. In the IBD group, LC3B expression significantly increased. Normal, normal group; control, control group; IBD, inflammatory bowel disease group; DCN, decorin.

a small number of autophagosomes with clear borders and undigested contents were observed in the NCM460 cells transfected with empty plasmid. In the NCM460 cells transfected with DCN expression plasmid, the number of autophagosomes increased significantly, which were vacuole-shaped with fully digested contents. These results indicate that autophagy was increased in NCM460 cells transfected with DCN expression plasmid. 
A

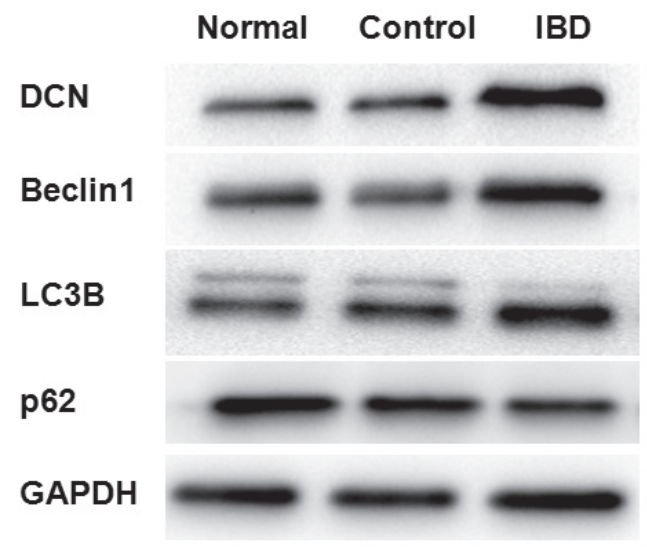

$\mathbf{B}$

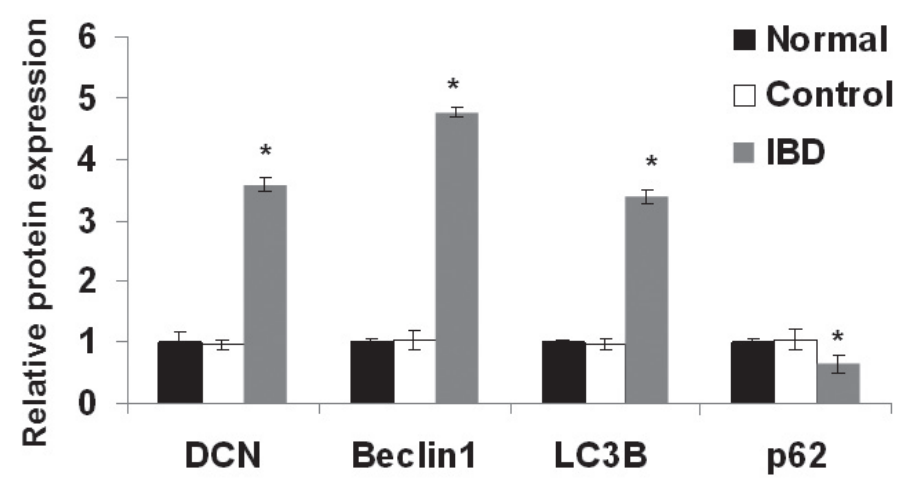

Figure 3. Expression of DCN and autophagy-associated proteins in the intestinal tissues detected using western blot analysis. (A) Representative results for DCN, beclin 1, LC3B, p62 and GAPDH are shown. GAPDH was used as an internal control. (B) Quantitative comparison of DCN, beclin 1, LC3B and p62 expression is shown. Data were derived from three independent experiments as in (A). The mean normalized optical density of DCN, beclin 1, LC3B and p62 protein bands relative to that of GAPDH bands from the same sample was calculated. Expression levels of DCN, beclin 1, LC3B and p62 are expressed as fold changes compared with the normal group. Data are expressed as mean \pm standard deviation. " $\mathrm{P}<0.05$ vs. the normal group Student's t-test. Normal, normal group; control, control group; IBD, inflammatory bowel disease group; DCN, decorin.

Decreased apoptosis in NCM460 cells transfected with DCN expression plasmid. As mentioned above, DCN expression was positively associated with the expression of autophagy-associated proteins. Under stress, autophagy can provide energy and protect cells (21). To investigate whether DCN protects the cells by regulating autophagy, NCM460 cells were transfected with DCN expression plasmid, cultured under oxygen glucose deprivation (OGD) conditions and treated with autophagy inhibitor 3-MA (5 $\mu \mathrm{M})$. Cell apoptosis was studied using an Annexin V apoptosis detection kit. As shown in Fig. 6, compared with the NCM460 cells without transfection or transfected with empty plasmid, apoptosis was significantly decreased in the cells transfected with DCN expression plasmid. Furthermore, the autophagy inhibitor 3-MA attenuated the effects of DCN overexpression on apoptosis.

\section{Discussion}

IBD is an autoimmune disease involving numerous inflammatory factors (22). It has been reported that inflammation induces autophagy, which degrades damaged organelles to provide energy and protect cells from apoptosis (23). It was thus hypothesized that autophagy may protect intestinal cells during the development of IBD. DCN is capable of negatively regulating the proliferation of tumor cells (24). In recent years, the effects of DCN on the occurrence and development of inflammation have aroused much attention. DCN may regulate the expression of a number of genes and is closely associated with inflammatory signaling pathways (25). In the present study, the expression of DCN as well as autophagy-associated proteins in IBD was examined for the first time, to the best of our knowledge. The correlation of DCN with autophagy and biological functions was also examined.

TNBS is able to induce colitis in mice with a high success rate, low mortality and pathological features similar to those of IBD in humans (26). Thus, in this study, an IBD mouse model produced by the intrarectal injection of TNBS was used. Immunohistochemical assays showed increased expression of DCN as well as autophagy-associated proteins in the intestinal tissues of the IBD mice. These results were further confirmed by western blot analysis. It appears that during the development of IBD, increased DCN expression is associated with autophagy and DCN participates in the regulation of autophagy. To test this hypothesis, NCM460 cells were transfected with DCN expression plasmid. The results showed significantly increased expression of autophagy-associated proteins and increased amounts of autophagosomes in the NCM460 cells with DCN overexpression. Furthermore, under OGD conditions, the apoptosis of NCM460 cells transfected with DCN expression plasmid was significantly lower than that 


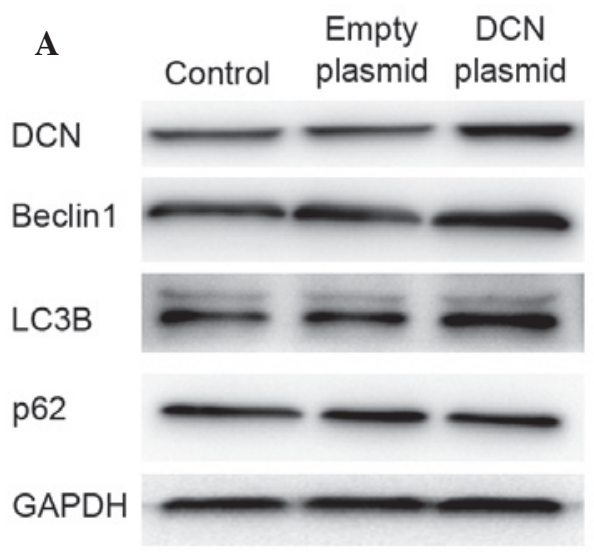

B

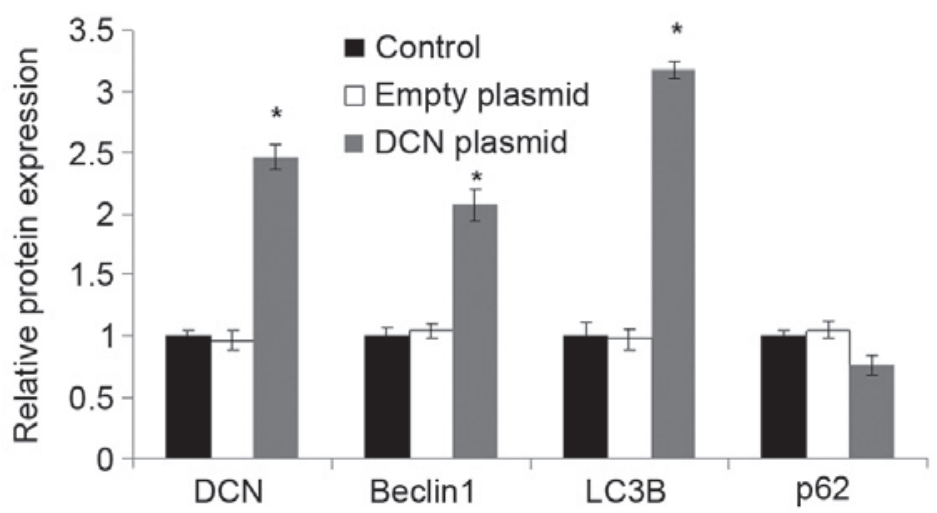

Figure 4. Increased expression of autophagy-associated proteins in NCM460 cells transfected with DCN expression plasmid. (A) Expression of DCNand autophagy-associated proteins was detected using western blot analysis. Representative results of DCN, beclin 1, LC3B, p62 and GAPDH are shown. GAPDH was used as an internal control. (B) Quantitative comparison of DCN, beclin 1, LC3B and p62 expression. Data were derived from three independent experiments as in (A). The mean normalized optical density of DCN, beclin 1, LC3B and p62 protein bands relative to that of GAPDH bands from the same sample was calculated. Expression levels of DCN, beclin 1, LC3B and p62 are expressed as fold changes compared with the normal group. Data are expressed as mean \pm standard deviation. "P<0.05 vs. the control group (Student's t-test). Control, NCM460 cells without transfection; empty plasmid, NCM460 cells transfected with empty plasmid; DCN plasmid, NCM460 cells transfected with DCN expression plasmid; DCN, decorin.

A
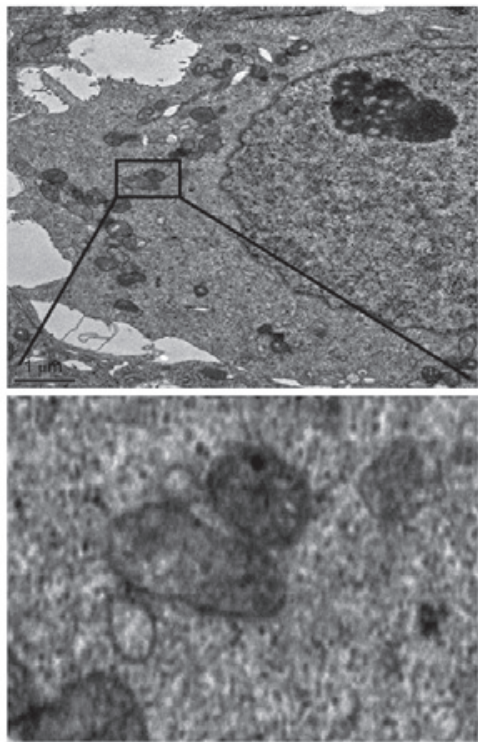

B

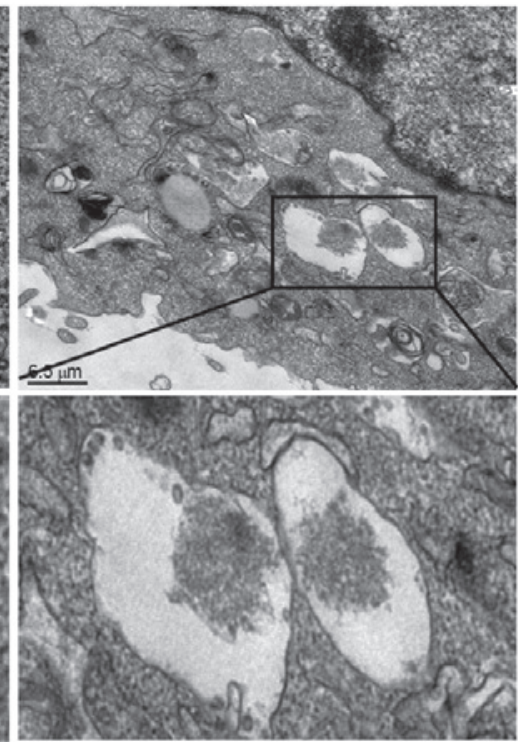

Figure 5. Increased autophagy in NCM460 cells transfected with DCN expression plasmid. Intracellular autophagosomes were observed using transmission electron microscopy (magnification, x200) and representative results are shown. (A) In the normal NCM460 cells transfected with empty plasmid, a small amount of autophagosomes are observed (upper image). Partial enlargement shows that the borders of the autophagosomes were clear and there were undigested contents in these autophagosomes (lower image). (B) In the NCM460 cells transfected with DCN expression plasmid, the number of autophagosomes increased significantly (upper image). Partial enlargement shows that the autophagosomes were vacuole-shaped with fully digested contents (lower image). DCN, decorin. 
A
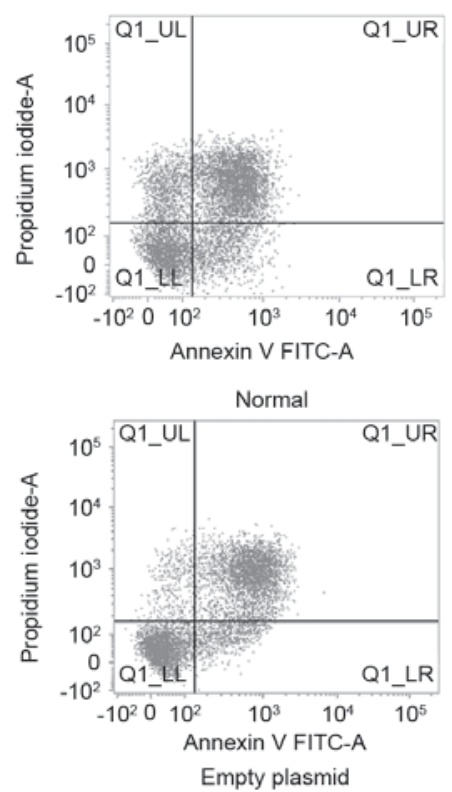

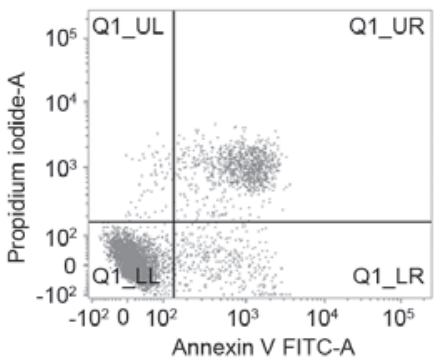

DCN plasmid

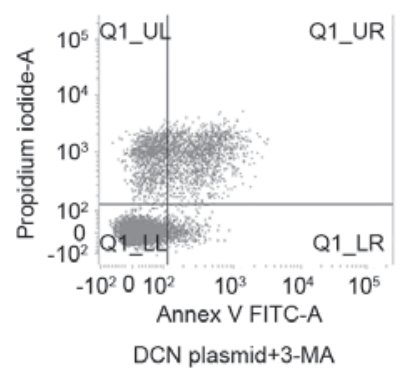

DCN plasmid+3-MA

B

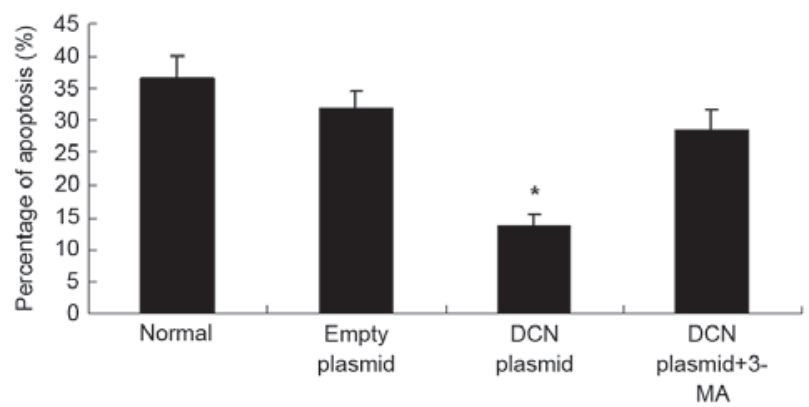

Figure 6. Decreased apoptosis in NCM460 cells transfected with DCN expression plasmid. NCM460 cells were cultured under oxygen glucose deprivation conditions. (A) Flow cytometric analysis of apoptosis using Annexin V and propidium iodide labeling. The experiments were performed thrice and representative results are shown. (B) Quantitative comparison of apoptosis. Data were derived from three independent experiments as in (A). Data are expressed as mean \pm standard deviation. ${ }^{*} \mathrm{P}<0.05$ vs. the normal group, Student's $\mathrm{t}$-test. Normal, NCM460 cells without transfection; empty plasmid, NCM460 cells transfected with empty plasmid; DCN plasmid, NCM460 cells transfected with DCN expression plasmid; DCN plasmid + 3-MA, NCM460 cells treated with DCN expression plasmid and treated with autophagy inhibitor 3-MA (5 $\mu \mathrm{M})$; FITC, fluorescein isothiocyanate; DCN, decorin; 3-MA, 3-methyladenine.

of the control group. In addition, autophagy inhibitor 3-MA treatment increased the apoptosis of these NCM460 cells with DCN overexpression.

In conclusion, these results indicate that $\mathrm{DCN}$ regulates autophagy and protects cells from apoptosis during the development of IBD. Thus, DCN may serve as a potential new target for IBD therapy.

\section{Acknowledgements}

This study was supported by the National High Technology Research and Development Program of China (grant no. 2012AA02A504) and Army Medical Science Youth Development Project (grant no. 13QNP185).

\section{References}

1. Agilli M, Aydin FN, Cayci T and Kurt YG: Assessment of active mucosal inflammation in IBD patients in clinical remission. J Gastrointestin Liver Dis 23: 462-463, 2014.

2. Dunkin D, Mehandru S and Colombel JF: Immune cell therapy in IBD. Dig Dis 32 (Suppl 1): S61-S66, 2014.
3. Hildner K, Punkenburg E, Abendroth B and Neurath MF: Immunopathogenesis of IBD: Batf as a Key Driver of Disease Activity. Dig Dis 34 (Suppl 1): 40-47, 2016.

4. De Salvo C, Ray S and Pizarro TT: Mechanisms and models for intestinal fibrosis in IBD. Dig Dis 32 (Suppl 1): S26-S34, 2014.

5. Latella G, Di Gregorio J, Flati V, Rieder F and Lawrance IC: Mechanisms of initiation and progression of intestinal fibrosis in IBD. Scand J Gastroenterol 50: 53-65, 2015.

6. Bryant RV, Brain O and Travis SP: Conventional drug therapy for inflammatory bowel disease. Scand J Gastroenterol 50: 90-112, 2015.

7. Chen M, Yi F, Zhou F, Huang M, Li J, Yan W, Li L and Xia B: Risk factors for initial surgery in patients with Crohn's disease in Central China. Surg Today 45: 1002-1008, 2015.

8. Connelly TM, Koltun WA, Sangster W, Berg AS, Hegarty JP, Harris L III, Deiling S and Stewart DB: An interleukin-4 polymorphism is associated with susceptibility to Clostridium difficile infection in patients with inflammatory bowel disease: Results of a retrospective cohort study. Surgery 156: 769-774, 2014.

9. Katz LH, Kopylov U, Fudim E, Yavzori M, Picard O, Ungar B, Eliakim R, Ben-Horin S and Chowers Y: Expression of IL-2, IL-17 and TNF-alpha in patients with Crohn's disease treated with anti-TNF antibodies. Clin Res Hepatol Gastroenterol 38: 491-498, 2014.

10. Zatorski H, Marynowski M and Fichna J: Is insulin-like growth factor 1 (IGF-1) system an attractive target inflammatory bowel diseases? Benefits and limitation of potential therapy. Pharmacol Rep 68: 809-815, 2016. 
11. Nata T, Fujiya M, Ueno N, Moriichi K, Konishi H, Tanabe H, Ohtake T, Ikuta K and Kohgo Y: MicroRNA-146b improves intestinal injury in mouse colitis by activating nuclear factor-kB and improving epithelial barrier function. J Gene Med 15: 249-260, 2013

12. Chen JY, Hour TC, Yang SF, Chien CY, Chen HR, Tsai KL, Ko JY and Wang LF: Autophagy is deficient in nasal polyps: Implications for the pathogenesis of the disease. Int Forum Allergy Rhinol 5: 119-123, 2015.

13. Liu H, He Z and Simon HU: Protective role of autophagy and autophagy-related protein 5 in early tumorigenesis. J Mol Med (Berl) 93: 159-164, 2015.

14. Annese V, Duricova D, Gower-Rousseau C, Jess T and Langholz E: Impact of New Treatments on Hospitalisation, Surgery, Infection, and Mortality in IBD: A Focus Paper by the Epidemiology Committee of ECCO. J Crohns Colitis 10: 216-225, 2016.

15. Murthi P, van Zanten DE, Eijsink JJ, Borg AJ, Stevenson JL, Kalionis B, Chui AK, Said JM, Brennecke SP and Erwich JJ: Decorin expression is decreased in first trimester placental tissue from pregnancies with small for gestation age infants at birth. Placenta 45: 58-62, 2016.

16. Nemani N, Santo L, Eda H, Cirstea D, Mishima Y, Patel C, O'Donnell E, Yee A and Raje N: Role of decorin in multiple myeloma (MM) bone marrow microenvironment. J Bone Miner Res 30: 465-470, 2015.

17. Esmaeili M, Berry M, Logan A and Ahmed Z: Decorin treatment of spinal cord injury. Neural Regen Res 9: 1653-1656, 2014.

18. Kasamatsu A, Uzawa K, Minakawa Y, Ishige S, Kasama H, Endo-Sakamoto Y, Ogawara K, Shiiba M, Takiguchi Y and Tanzawa H: Decorin in human oral cancer: A promising predictive biomarker of S-1 neoadjuvant chemosensitivity. Biochem Biophys Res Commun 457: 71-76, 2015.

19. Araki K, Wakabayashi H, Shintani K, Morikawa J, Matsumine A, Kusuzaki K, Sudo A and Uchida A: Decorin suppresses bone metastasis in a breast cancer cell line. Oncology 77: 92-99, 2009.
20. Reed CC, Waterhouse A, Kirby S, Kay P, Owens RT,McQuillan DJ and Iozzo RV: Decorin prevents metastatic spreading of breast cancer. Oncogene 24: 1104-1110, 2005.

21. Obba S, Hizir Z, Boyer L, Selimoglu-Buet D, Pfeifer A, Michel G, Hamouda MA, Gonçalvès D, Cerezo M, Marchetti S, et al: The PRKAA1/AMPK $\alpha 1$ pathway triggers autophagy during CSF1-induced human monocyte differentiation and is a potential target in CMML. Autophagy 11: 1114-1129, 2015.

22. Cheng X, Zhang X, Su J, Zhang Y, Zhou W, Zhou J, Wang C, Liang H, Chen X, Shi R, et al: miR-19b downregulates intestinal SOCS3 to reduce intestinal inflammation in Crohn's disease. Sci Rep 5: 10397, 2015.

23. Zeng TS, Liu FM, Zhou J, Pan SX, Xia WF and Chen LL: Depletion of Kupffer cells attenuates systemic insulin resistance, inflammation and improves liver autophagy in high-fat diet fed mice. Endocr J 62: 615-626, 2015.

24. Mauviel A, Santra M, Chen YQ, Uitto J and Iozzo RV: Transcriptional regulation of decorin gene expression. Induction by quiescence and repression by tumor necrosis factor-alpha. J Biol Chem 270: 11692-11700, 1995.

25. Schaefer L, Macakova K, Raslik I, Micegova M, Gröne HJ, Schönherr E, Robenek H, Echtermeyer FG, Grässel S, Bruckner $\mathrm{P}$, et al: Absence of decorin adversely influences tubulointerstitial fibrosis of the obstructed kidney by enhanced apoptosis and increased inflammatory reaction. Am J Pathol 160: 1181-1191, 2002.

26. Tomasello G, Sinagra E, Raimondo D, Palumbo VD, Puleio R, Cottone M, Damiani P, Traina G, Abruzzo A, Damiani F, et al: Validation of a modified model of TNBS-induced colitis in rats. How to induce a chemical colitis in rats. Acta Biomed 86: 92-96, 2015. 